Universidade Tecnológica Federal do Paraná - UTFPR

Campus Ponta Grossa - Paraná - Brasil

ISSN: $1981-3686 /$ v. 05, n. $02:$ p. $516-523,2011$

D.O.I.: $10.3895 / \mathrm{S} 1981-36862011000200003$
Revista Brasileira de Tecnologia

Agroindustrial

\title{
COMPORTAMENTO DA ACIDEZ EM MANGA IN NATURA ARMAZENADA EM ATMOSFERA CONTROLADA
}

\section{BEHAVIOR OF ACIDITY IN MANGO IN NATURE STORED IN CONTROLLED ATMOSPHERE}

\author{
Esther Maria Barros de Albuquerque ${ }^{1}$, Dyego da Costa Santos ${ }^{2}$, Emanuel Neto Alves de Oliveira ${ }^{3}$, \\ Francisco de Assis Cardoso Almeida ${ }^{4}$, Humberto Silva ${ }^{5}$ \\ 1,2,3,4 Universidade Federal de Campina Grande - UFCG - E-mail: esther_barros@ hotmail.com \\ ${ }^{5}$ Universidade Estadual da Paraíba - UEPB - E-mail: humbertosilva@ ccbs.uepb.edu.br
}

\begin{abstract}
Resumo
O objetivo do estudo foi verificar o comportamento da acidez em mangas (Mangifera indica $L$.), da variedade Haden, armazenadas nas atmosferas modificadas: sem embalagem (SE), plástico perfurado $(P P)$ e filme plástico $(F P)$ nas temperaturas de 5 e $10^{\circ} \mathrm{C}$ e temperatura ambiente $( \pm 26$ ${ }^{\circ} \mathrm{C}$ ) de Campina Grande, PB. Cinqüenta e quatro mangas foram selecionadas aleatoriamente (de acordo com a coloração da casca) para composição dos tratamentos e acondicionados em bandejas de tereftalato, e descritos como segue: não revestidos com filme plástico (SE); revestidos externamente com filme plástico não perfurado $(F P)$ e revestidos externamente com plástico perfurado (PP). Em seguida as mangas foram armazenadas em condições ambientais de Campina Grande, $P B\left( \pm 26{ }^{\circ} \mathrm{C}\right)$ e em câmaras frias nas temperaturas de 5 e $10{ }^{\circ} \mathrm{C}$. No decorrer do armazenamento efetuaram-se análises de acidez em períodos de 7 dias, de acordo com a metodologia descrita por Cecchi (2003). As mangas acondicionadas em embalagem de filme plástico (FP) a $5{ }^{\circ} \mathrm{C}$ conservaram o maior teor de acidez. Os teores de acidez sofreram variações, sendo revelado o maior teor no $1^{\circ}$ dia de armazenagem à temperatura de $5{ }^{\circ} \mathrm{C}$, enquanto o menor teor foi verificado à temperatura ambiente de $\pm 26^{\circ} \mathrm{C}$ a partir do $28^{\circ}$ dia de acondicionamento. Os resultados obtidos indicam a temperatura e a atmosfera interferem no teor de acidez.
\end{abstract}

Palavras-chave: Mangifera indica L., armazenagem, embalagem, acidez.

\section{Introdução}

A manutenção da qualidade dos frutos deve-se a técnicas de armazenamento pós-colheita que reduzem as taxas respiratórias e retardam o amadurecimento e prevenção de desordem. A perda de água e a decomposição natural do fruto podem ser evitadas pelo abaixamento da temperatura e modificação da atmosfera ou mesmo à combinação de ambos, imediatamente após a colheita. 
No armazenamento sob atmosfera modificada (AM), a atmosfera do ambiente é alterada pelo uso de filmes plásticos ou recobrimentos comestíveis, permitindo que a concentração de $\mathrm{CO}_{2}$ aumente e a de $\mathrm{O}_{2}$ diminua decorrente da respiração do fruto. Neste sistema, as concentrações dos gases não são controladas, variando com o tempo, temperatura, permeabilidade do filme e atividade respiratória do produto. O filme plástico deve apresentar permeabilidade seletiva adequada à entrada de $\mathrm{O}_{2}$ e saída de $\mathrm{CO}_{2}$, de modo que o produto não entre em anaerobiose ou processo de fermentação (CHITARRA; CHITARRA, 2005).

Várias pesquisas já foram desenvolvidas para avaliar o comportamento da acidez em mangas armazenadas em atmosfera modificada: Souza et al. (2002) estudaram a influência do armazenamento refrigerado em associação com atmosfera modificada por filmes plásticos na qualidade de mangas 'Tommy Atkins'; Pfaffenbach et al. (2003) verificaram o efeito da atmosfera modificada e da refrigeração na conservação pós-colheita de manga espada vermelha; Jerônimo et al. (2007) avaliaram a qualidade de mangas 'Tommy Atkins' armazenadas sob atmosfera modificada; Rodrigues et al. (2008) investigaram a vida útil de fatias de manga da variedade 'Tommy Atkins' armazenadas em embalagem com atmosfera modificada passiva; Ribeiro et al. (2009) estudaram o uso de revestimentos à base de dextrina na conservação pós-colheita de manga 'Tommy Atkins'. Santos et al. (2011) pesquisaram o efeito do tratamento hidrotérmico e diferentes revestimentos na conservação pós-colheita de mangas 'Tommy Atkins'.

Diante do exposto, o objetivo do estudo foi verificar o comportamento da acidez em mangas (Mangifera indica L.), da variedade Haden, armazenadas nas atmosferas modificadas: sem embalagem (SE), plástico perfurado (PP) e filme plástico (FP) nas temperaturas de 5 e $10{ }^{\circ} \mathrm{C}$ e temperatura ambiente $\left( \pm 26^{\circ} \mathrm{C}\right)$ de Campina Grande, PB.

\section{Material e Métodos}

Local de realização da pesquisa

O estudo foi desenvolvido no Laboratório de Armazenamento e Processamento de Produtos Agrícolas (LAPPA) da Unidade Acadêmica de Engenharia Agrícola (UAEAg) da Universidade Federal de Campina Grande (UFCG) em parceria com a Universidade Estadual da Paraíba (UEPB), na cidade de Campina Grande, PB.

\section{Matéria prima}

Foram adquiridas 54 mangas (Mangifera indica L.) da variedade Haden safra 2007, no comercio atacadista/varejista da Empresa Paraibana de Abastecimento e Serviços Agrícolas (EMPASA) na cidade de Campina Grande, PB. 
A seleção foi baseada no desenvolvimento da cor da casca durante o amadurecimento da manga (Mangifera indica L.), considerando o trabalho de Pfaffenbach et al. (2003). Na presente pesquisa o amarelo foi substituído por arroxeado, em virtude do tipo de manga trabalhada (Figura $1)$.

Figura 1 - Desenvolvimento da cor da casca da manga durante o seu amadurecimento.

\begin{tabular}{|l|l|l|}
\hline \multicolumn{3}{|c|}{ Desenvolvimento da cor da casca durante o amadurecimento do fruto } \\
\hline $\mathbf{2}$ & & \\
\hline 3 & & A cor normal do fruto depois da colheita é principalmente verde escuro. \\
\hline $\mathbf{3}$ & & Quando a maturidade se faz presente, os frutos são mais verdes que amarelo. \\
\hline $\mathbf{4}$ & Os frutos apresentam quantidades iguais de verde e amarelo. \\
\hline $\mathbf{5}$ & & Nesta etapa o fruto são mais amarelo que verde. \\
\hline
\end{tabular}

Fonte: PFAFFENBACH et al., 2003

\section{Tratamento dos frutos}

Na unidade de comercialização os frutos foram pré-selecionados através de análise visual, de acordo com os atributos de qualidade como cor, uniformidade, grau de maturação (estádio 2, conforme Figura 1), ausência de injúrias ou doenças e massa (400-500 g).

Em seguida as amostras foram conduzidas ao Laboratório de Armazenamento de Processamento de Produtos Agrícolas (LAPPA) da Universidade Federal de Campina Grande, onde foram submetidas a uma nova seleção. As etapas de pré-lavagem, lavagem e sanitização foram executadas de acordo com as normas higiênico-sanitárias indicadas pelas Boas Práticas de Fabricação (BPF). Os frutos foram selecionados aleatoriamente para composição dos tratamentos e descritos como segue:

1. Frutos acondicionados em bandejas de polietileno tereftalato $(29 \times 16 \times 2 \mathrm{~cm})$, não revestidas com filme plástico, que corresponde ao grupo controle SE (Figura 2); 
2. Frutos acondicionados em bandejas de tereftalato $(29 \times 16 \times 2 \mathrm{~cm})$, revestidas externamente com filme plástico de cloreto de polivinila (PVC) esticável e autoaderente não perfurado, que corresponde ao grupo controle FP (Figura 2);

3. Frutos acondicionados em bandejas de tereftalato $(29 \times 16 \times 2 \mathrm{~cm})$, revestidas externamente com plástico perfurado, que corresponde ao grupo controle PP (Figura 2).

Figura 2 - Tratamentos utilizados na avaliação do comportamento da acidez em mangas da variedade Haden armazenadas em atmosfera modificada.

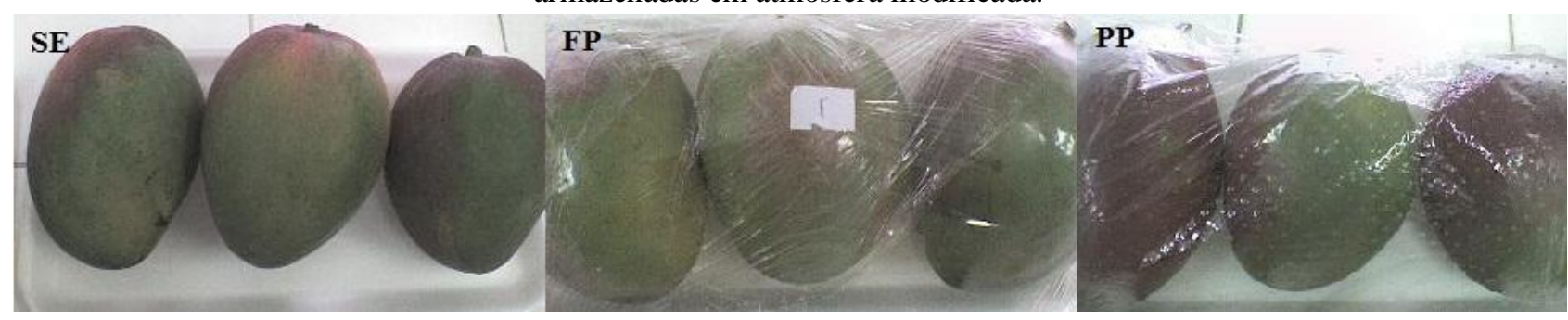

SE - Sem embalagem; FP - Filme plástico; PP - plástico perfurado.

Em seguida as mangas foram armazenadas em condições ambientais de Campina Grande, PB $\left( \pm 26{ }^{\circ} \mathrm{C}\right)$ e em câmaras frias nas temperaturas de 5 e $10{ }^{\circ} \mathrm{C}$, as quais continham um leitor de temperatura digital na parte externa.

\section{Determinação da acidez}

As mangas foram avaliados aos 0, 7, 14, 21, 28 e 35 dias de armazenamento. Na determinação da acidez titulável empregou-se a metodologia descrita por Cecchi (2003) e, os resultados expressos em mg. $100 \mathrm{~g}^{-1}$ de ácido cítrico.

Para a avaliação desse parâmetro, as mangas foram inicialmente pesadas e despolpadas com o auxilio de uma faca de aço inoxidável, com homogeneização em liquidificador doméstico.

\section{Análise dos dados}

As análises foram realizadas em quadruplicata, empregando-se a média para o parâmetro analisado. Após a análise de variância, quando constatada significância pelo teste $\mathrm{F}$, os tratamentos foram comparados através do teste Tukey ao nível de 5\% de significância.

Os tratamentos analisados foram: (1) temperatura $\left(5\right.$ e $10{ }^{\circ} \mathrm{C}$ e temperatura ambiente $( \pm 26$ ${ }^{\circ} \mathrm{C}$ ) de Campina Grande, PB); (2) embalagem (sem embalagem, filme plástico e plástico perfurado) e (3) tempo de armazenamento $\left(\mathrm{T}_{0}, \mathrm{~T}_{7}, \mathrm{~T}_{14}, \mathrm{~T}_{21}, \mathrm{~T}_{28}\right.$ e $\mathrm{T}_{35}$ dias $)$. 


\section{Resultados e Discussão}

A análise de variância da acidez obtida experimentalmente revelou valores de $\mathrm{F}$ altamente significativos para todos os fatores e suas interações (Tabela 1).

Tabela 1 - Análise de variância da Acidez da polpa da manga Haden submetida a três atmosferas por 35 dias em três diferentes temperaturas

\begin{tabular}{lcccc}
\hline \multicolumn{1}{c}{ Fonte de variação } & G.L. & S.Q. & Q.M. & F \\
\hline Temperatura(T) & 2 & 1974,74996 & 987,37498 & $2974,4358 * *$ \\
Atmosfera (Atm) & 2 & 321,77411 & 160,88706 & $484,6672 * *$ \\
Período de armazenamento(P) & 5 & 1213,67585 & 242,73517 & $731,2320 * *$ \\
T x Atm & 4 & 59,23707 & 14,80927 & $44,6125 * *$ \\
T x P & 10 & 657,37890 & 65,73789 & $198,0333 * *$ \\
Atm x P & 10 & 415,84105 & 41,58410 & $125,2708 * *$ \\
T x Atm x P & 20 & 447,59701 & 22,37985 & $67,4186 * *$ \\
Resíduo & 162 & 53,77650 & 0,33195 & \\
Total & 215 & 5144,03046 & & \\
\hline
\end{tabular}

Nível de diferença significativa entre as variáveis de entrada e saída

${ }^{\mathrm{ns}}$ não significativo, * significativo a $5 \%$ e ** significativo a $1 \%$ de probabilidade, pelo teste $\mathrm{F}$.

Verifica-se na Tabela 2 a interação temperatura $x$ atmosfera. Observa-se que a manga acondicionada na atmosfera filme plástico (FP) apresentou maior percentual de acidez quando a temperatura foi igual a $5{ }^{\circ} \mathrm{C}\left(12,2708 \mathrm{mg} \cdot 100^{-1}\right)$, diferindo estatisticamente das mangas armazenadas nas atmosferas plástico perfurado (PP) e sem embalagem (SE). O menor teor de acidez foi encontrado na atmosfera SE $\left(2,8275 \mathrm{mg} .100^{-1}\right)$ na temperatura ambiente de $\pm 26{ }^{\circ} \mathrm{C}$.

Quando as mangas foram acondicionadas na atmosfera SE, PP e FP, os maiores percentuais de acidez foram revelados na temperatura de $5^{\circ} \mathrm{C}\left(10,9771 ; 10,4563\right.$ e $12,2708 \mathrm{mg} .100^{-}$ ${ }^{1}$, respectivamente). Os menores teores de acidez foram encontrados na temperatura ambiente de $\pm 26^{\circ} \mathrm{C}$ para todas as atmosferas.

Tabela 2 - Influência da interação temperatura x atmosfera sobre o teor de Acidez presentes na manga Haden

\begin{tabular}{cccc}
\hline & & & \\
Temperatura & & Atmosfera $^{1}$ & \\
\cline { 2 - 4 } & SE & PP & FP \\
\hline Ambiente & $2,8275 \mathrm{cB}$ & $3,2092 \mathrm{cB}$ & $5,6983 \mathrm{cA}$ \\
$10{ }^{\circ} \mathrm{C}$ & $6,4938 \mathrm{bB}$ & $4,5238 \mathrm{bC}$ & $8,8242 \mathrm{bA}$ \\
$5{ }^{\circ} \mathrm{C}$ & $10,9771 \mathrm{aB}$ & $10,4563 \mathrm{aC}$ & $12,2708 \mathrm{aA}$ \\
\hline
\end{tabular}

${ }^{1}$ Médias seguidas da mesma letra, na coluna, não diferem estaticamente, a 5\% de probabilidade pelo teste de Tukey; *SE (Sem Embalagem); PP (Plástico Perfurado); FP (Filme plástico). 
Observa-se na Tabela 3 que a medida que avança o tempo de armazenamento houve redução nos teores de acidez em todas as temperaturas estudadas. Comportamento semelhante foi observado por O’Hare (1995) para as mangas Kensington, onde os teores de acidez titulável diminuíram conforme o amadurecimento dos frutos. O teor de ácidos orgânicos geralmente diminui durante o processo de maturação, devido à oxidação dos ácidos no ciclo dos tricarboxílicos em decorrência da respiração.

Tabela 3 - Influência da interação Temperatura x Período de Armazenamento sobre o teor de Acidez presentes na manga Haden

\begin{tabular}{ccccccc}
\hline \multirow{2}{*}{ Temperatura } & \multicolumn{7}{c}{ Período de Armazenamento (dias) ${ }^{\mathbf{1}}$} \\
\cline { 2 - 7 } & 0 & 7 & 14 & 21 & 28 & 35 \\
\cline { 2 - 7 } & $9,9500 \mathrm{cA}$ & $4,2642 \mathrm{cC}$ & $7,3208 \mathrm{bB}$ & $1,9350 \mathrm{cD}$ & $0,0000 \mathrm{cE}$ & $0,0000 \mathrm{cE}$ \\
\hline Ambiente & $11,3808 \mathrm{bA}$ & $7,7917 \mathrm{bB}$ & $7,1800 \mathrm{bC}$ & $7,9225 \mathrm{bB}$ & $4,1517 \mathrm{bD}$ & $1,2567 \mathrm{bE}$ \\
$5^{\circ} \mathrm{C}$ & $12,0433 \mathrm{aB}$ & $13,2750 \mathrm{aA}$ & $10,9575 \mathrm{aC}$ & $9,5467 \mathrm{aD}$ & $9,3500 \mathrm{aD}$ & $7,2358 \mathrm{aB}$ \\
\hline
\end{tabular}

${ }^{1}$ Médias seguidas da mesma letra, na coluna, não diferem estaticamente, a 5\% de probabilidade pelo teste de Tukey

Na Tabela 4 encontram-se os resultados referentes à influência da interação atmosfera $\mathrm{x}$ período de armazenamento sobre o teor de acidez.

Observa-se que as mangas armazenadas nas atmosferas SE, PP e FP apresentaram redução no teor de acidez com o decorrer do período de armazenamento, sendo este maior em FP e estatisticamente igual a SE. Verifica-se que o PP obteve os menores teores de acidez encontrados na manga Haden.

De acordo com Chitarra e Chitarra (2000), estes resultados assemelham-se ao comportamento da laranja, decréscimo até o completo amadurecimento.

Tabela 4 - Influência da interação Atmosfera x Período de Armazenamento sobre o teor de Acidez presentes na manga Haden.

\begin{tabular}{ccccccc}
\hline \multirow{2}{*}{ Atmosfera } & \multicolumn{7}{c}{ Período de Armazenamento (Dias) $^{\mathbf{1}}$} \\
\cline { 2 - 7 } & 0 & 7 & 14 & 21 & 28 & 35 \\
\cline { 2 - 7 } SE & $12,8483 \mathrm{aA}$ & $7,8100 \mathrm{bB}$ & $5,7258 \mathrm{cC}$ & $5,5983 \mathrm{bC}$ & $4,3133 \mathrm{bD}$ & $4,3008 \mathrm{bD}$ \\
PP & $8,2150 \mathrm{bB}$ & $6,3742 \mathrm{cC}$ & $9,4958 \mathrm{bA}$ & $3,6817 \mathrm{cE}$ & $3,4992 \mathrm{cE}$ & $5,1125 \mathrm{aD}$ \\
FP & $12,3108 \mathrm{aA}$ & $11,1467 \mathrm{aB}$ & $10,2367 \mathrm{aC}$ & $10,1242 \mathrm{aC}$ & $5,6892 \mathrm{aD}$ & $4,0792 \mathrm{bE}$ \\
\hline
\end{tabular}

${ }^{1}$ Médias seguidas da mesma letra, na coluna, não diferem estaticamente, a 5\% de probabilidade pelo teste de Tukey; *SE (Sem Embalagem); PP (Plástico Perfurado); FP (Filme plástico).

Segundo Krishnamurthy e Subramanyam (1973) e Alaniswamy et al. (1996) o decréscimo de acidez, contido em grande quantidade nos frutos verdes, dá-se de modo muito lento, podendo 
ocorrer prejuízos no momento da colheita, já que em alguns casos, o fruto apresenta-se completamente amarelado, porém ainda com a polpa ácida.

Pode-se acrescentar que é de grande importância não só a queda do conteúdo ácido da manga, mas também o equilíbrio conveniente entre teor de açúcar e acidez, para que o produto obtido seja o melhor possível em termos de qualidade de consumo (MEDLICOTT; BHOGOL; REYNOLDS, 1986).

\title{
4. Conclusão
}

Observou-se que as mangas acondicionadas em bandejas de tereftalato revestidas com filme plástico (FP) a $5^{\circ} \mathrm{C}$ conservaram o maior teor de acidez, enquanto os menores teores foram encontrados na atmosfera sem embalagem (SE) à temperatura ambiente de $\pm 26^{\circ} \mathrm{C}$.

No decorrer do armazenamento e da maturação, os teores de acidez sofreram variações, sendo revelado o maior teor no $1^{\circ}$ dia de armazenagem à temperatura de $5{ }^{\circ} \mathrm{C}$, enquanto o menor teor foi verificado à temperatura ambiente de $\pm 26^{\circ} \mathrm{C}$ a partir do $28^{\circ}$ dia.

Os resultados obtidos indicam que tanto a temperatura quanto a atmosfera interferem no teor de acidez.

\begin{abstract}
The aim of this study was to investigate the behavior of acidity in mangoes (Mangifera indica L.), variety Haden, stored in modified atmospheres: no packaging (SE), perforated plastic (PP) and plastic film $(P F)$ at temperatures $5,10{ }^{\circ} \mathrm{C}$ and ambient $\left( \pm 26^{\circ} \mathrm{C}\right)$ of Campina Grande, PB. Fifty four mangas were selected randomly (according to the peel of the fruit) to treatment composition, packed in trays terephthalate, and described as follows: without package (SE); externally coated with non-perforated plastic film (PF) and coated externally with perforated plastic (PP). Then the mangos were stored in environmental conditions of Campina Grande, $P B\left( \pm 26^{\circ} \mathrm{C}\right)$ and cold chambers at temperatures of 5 and $10^{\circ} \mathrm{C}$. During storage were conducted acid analysis, in periods of seven days, according to the method described by Cecchi (2003). The Mangoes packed in plastic film $(P F)$ at $5^{\circ} \mathrm{C}$ maintained the highest level of acidity. Acidity levels have varied, and revealed the highest level on day 1 of storage at $5^{\circ} \mathrm{C}$, while the lowest content was observed at room temperature of $\pm 26^{\circ}$ C from $28^{\circ}$ day. The results indicate that temperature and atmosphere affect the acidity.
\end{abstract}

Key-words: Mangifera indica L., storage, packaging, acidity.

\section{Referências}

ALANISWAMY, M.; S WAMINATHAN, K. Cultivation of Pleurotus spp. On various agro-residues. Food Chemistry, v. 55, p. 139-144, 1996. http://dx.doi.org/10.1016/0308-8146(95)00079-8

CECCHI, H. M. Fundamentos teóricos e práticos em analise de alimentos. 2 ed. rev., Campinas: Editora UNICAMP, 2003, 207p. 
CHITARRA, A. B.; CHITARRA, M. I. F. Fisiologia pós-colheita de frutos e hortaliças. Lavras: FAEPE, 2000.

CHITARRA, M. I. F.; CHITARRA, A. B. Pós-colheita de frutos e hortaliças: fisiologia e manuseio. 2.ed. Lavras: UFLA, 2005.

JERÔNIMO, E. M.; BRUNINI, M. A.; ARRUDA, M. C.; CRUZ, J. C. S.; GAVA, G. J. C.; SILVA, M. A. Qualidade de mangas 'Tommy Atkins' armazenadas sob atmosfera modificada. Ciência e Agrotecnologia, v. 31, n. 4, p. 11221130, 2007. http://dx.doi.org/10.1590/S1413-70542007000400027

KRISHNAMURTHY, S.; SUBRAMANYAM, H. Pre and post-harvest physiology of de mango fruit: a review. Tropical Science, Oxford, v. 15, p. 167-193, 1973.

MEDLICOTT, A. P.; BHOGOL, M.; REYNOLDS, S. B. Changes in peel pigmentation during ripening of mango fruit (Mangifera indica var. Tommy Atkins). Annals of Applied Biology, London, v. 109, n. 3, p. 651-656, 1986. http://dx.doi.org/10.1111/j.1744-7348.1986.tb03222.x

O'HARE, T. J. Effect of ripening temperature on quality and compositional changes of mango (Mangifera indica L.) cv. Kensington. Australian Journal of Experimental Agricultural, Victoria, v. 35, n. 2, p. 259-63, 1995. http://dx.doi.org/10.1071/EA9950259

PFAFFENBACH, L. B.; CASTRO, J. V.; CARVALHO, C. R. L.; ROSSETTO, C. J. Efeito da atmosfera modificada e da refrigeração na conservação pós-colheita de manga espada vermelha. Revista Brasileira de Fruticultura, v. 25 , n. 3, p. 410-413, 2003. http://dx.doi.org/10.1590/S0100-29452003000300012

RIBEIRO, T. P.; LIMA, M. A. C.; TRINDADE, D. C. G.; SANTOS, A. C. N.; AMARIZ, A. Uso de revestimentos à base de dextrina na conservação pós-colheita de manga ‘Tommy Atkins'. Revista Brasileira de Fruticultura, v. 31 , n. 2, p. 343-351, 2009. http://dx.doi.org/10.1590/S0100-29452009000200008

RODRIGUES, L. K.; PEREIRA, L. M.; FERRARI, C. C.; SARANTÓPOULOS, C. I. G. L.; HUBINGER, M. D. Vida útil de fatias de manga armazenadas em embalagem com atmosfera modificada passiva. Ciência e Tecnologia de Alimentos, v. 28, supl., p. 271-278, 2008

SANTOS, A. E. O.; GRAVINA, G. A.; BERBERT, P. A.; ASSIS, J. S.; BATISTA, P. F.; SANTOS, O. O. Efeito do tratamento hidrotérmico e diferentes revestimentos na conservação pós-colheita de mangas 'Tommy Atkins'. Revista Brasileira de Ciências Agrárias, v. 6, n. 1, p. 140-146, 2011.

SOUSA, J. P.; PRAÇA, E. F.; ALVES, R. E.; NETO, F.; DANTAS, F. F. Influência do armazenamento refrigerado em associação com atmosfera modificada por filmes plásticos na qualidade de mangas 'Tommy Atkins'. Revista Brasileira de Fruticultura, v. 24, n. 3, p. 665-668, 2002. http://dx.doi.org/10.1590/S0100-29452002000300023

Submetido em 19 ago. 2010; Revisão submetida pelos autores em 25 set. 2011; Aceito para publicação em 13 dez.2011. 